\title{
Control Glaucomatous
}
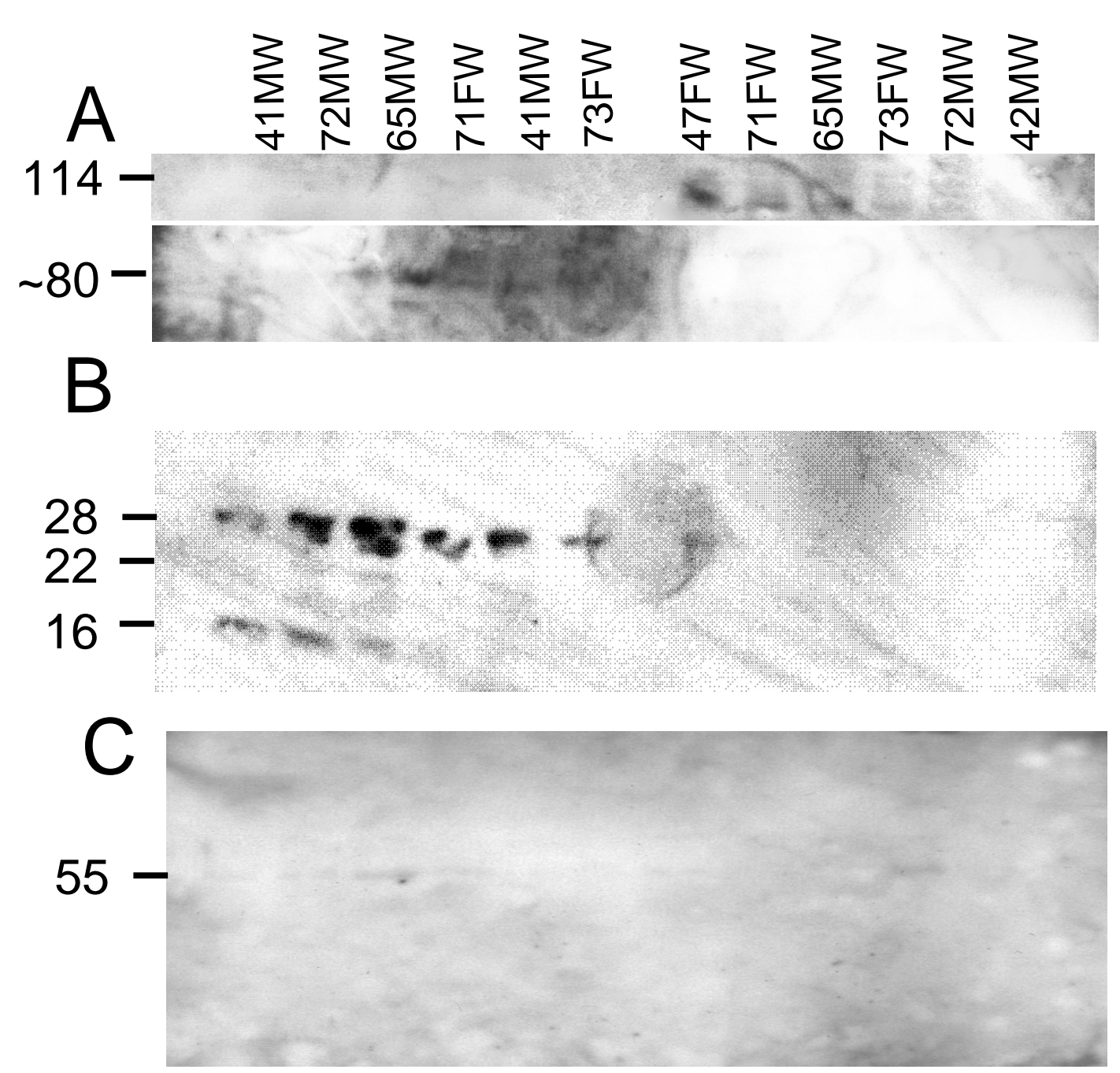

Supplementary Figure S1. Western analysis of trabecular meshwork proteins. TM tissue extract $(10 \mu \mathrm{g})$ was loaded on a 4-20\% gradient gel (Invitrogen, CA) after transfer on a PVDF, the blot was probed with antibodies. (A) rabbit polyclonal antibody against calpain-2 large subunit , (B) monoclonal antibody against calpain-1 small subunit, (C) rabbit polyclonal antibody against rabbit IgG. 


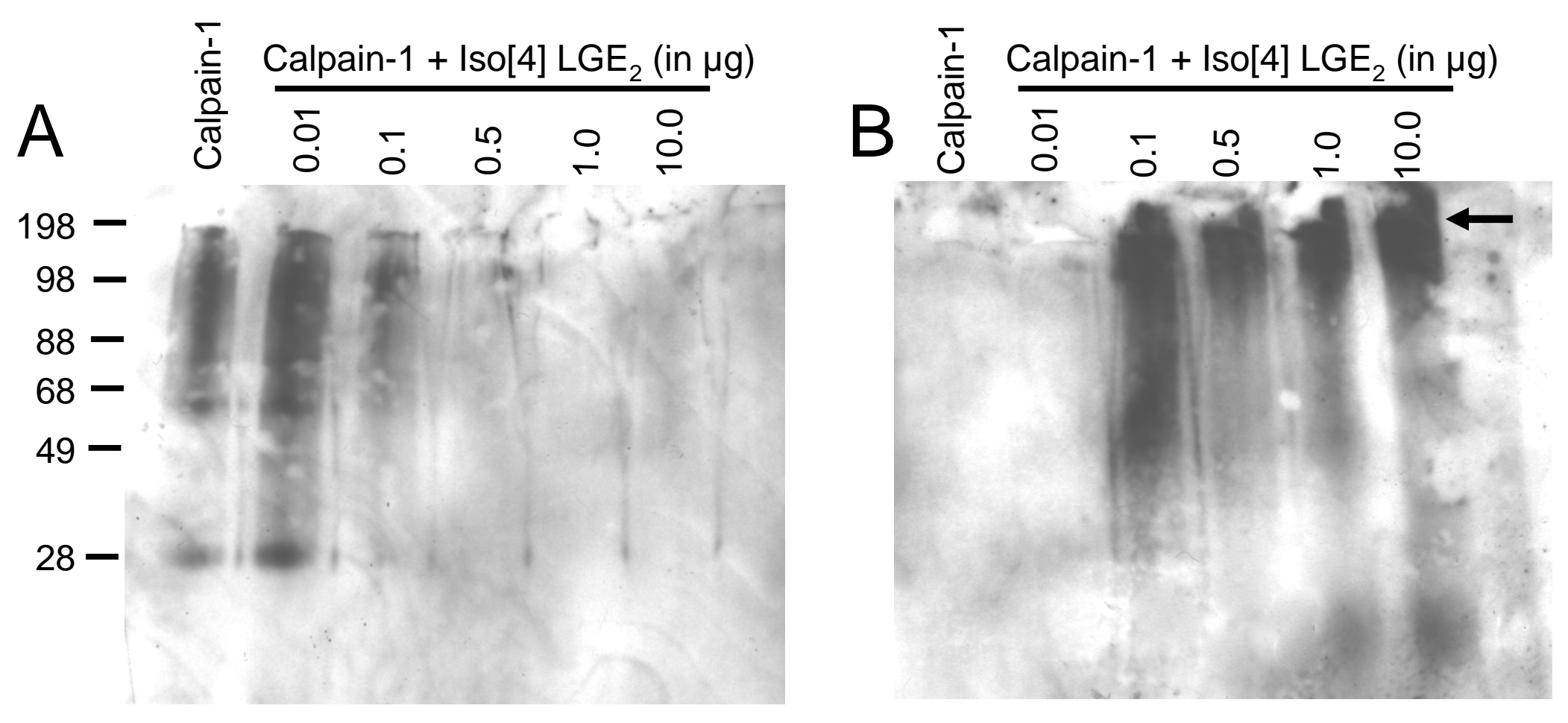

Supporting Figure S2. Formation of high molecular weight aggregate covalent adducts of calpain-1 and iso[4] $\mathrm{LGE}_{2}$. Western analyses for calpain-1 modification by iso[4]LGE $\mathrm{L}_{2}$ utilizing plugged variable field SDS-PAGE (A) probed with calpain-1 antibody. (B) probed with iso[4] $\mathrm{LGE}_{2}$ antibody. 
$\stackrel{\vec{J}}{\stackrel{-}{\sigma}}$ Purified digested Calpain-1

A

$\frac{O}{\sqrt{D}}$ (fragments)

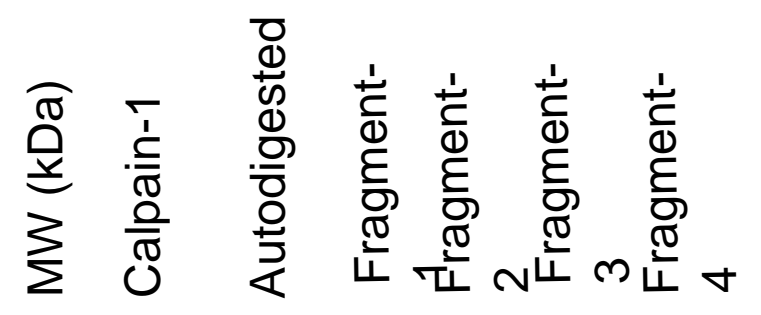

B
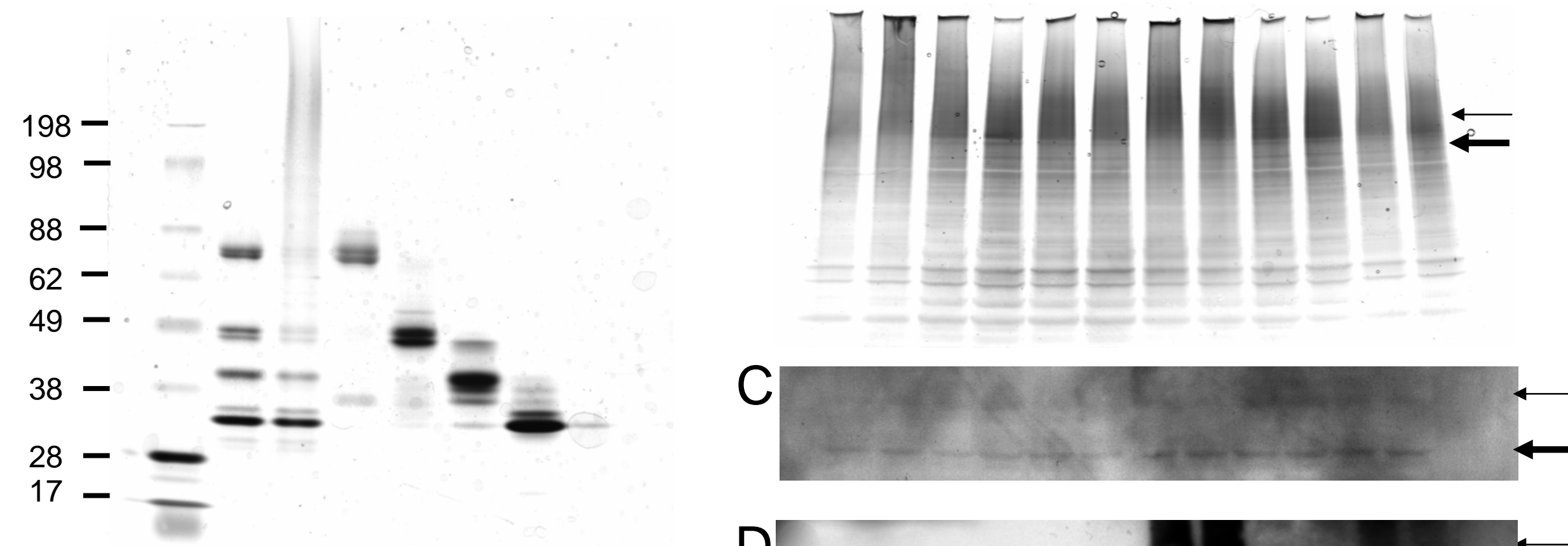

D

Supplemental Figure S3. (A) Purification of Calpain-1 fragments. About $2 \mu \mathrm{g}$ purified calpain-1 from human erythrocytes were subjected to silver staining M227 SILVER BULLit silver stain kit (Amresco, Solon, OH). Calpain-1, fragmented calpain-1 and iso[4]LGE ${ }_{2}$ modified calpain-1 have different in vitro ubiquination rates. Ubiquitination was performed using a kit (Boston biochemicals, MA) followed by electrophoretic separation using SDS-PAGE. (B) Silver stained gel. (C) Western analysis with anti-ubiquitin and (D) Western analysis using iso[4]LGE ${ }_{2}$ antibody. 


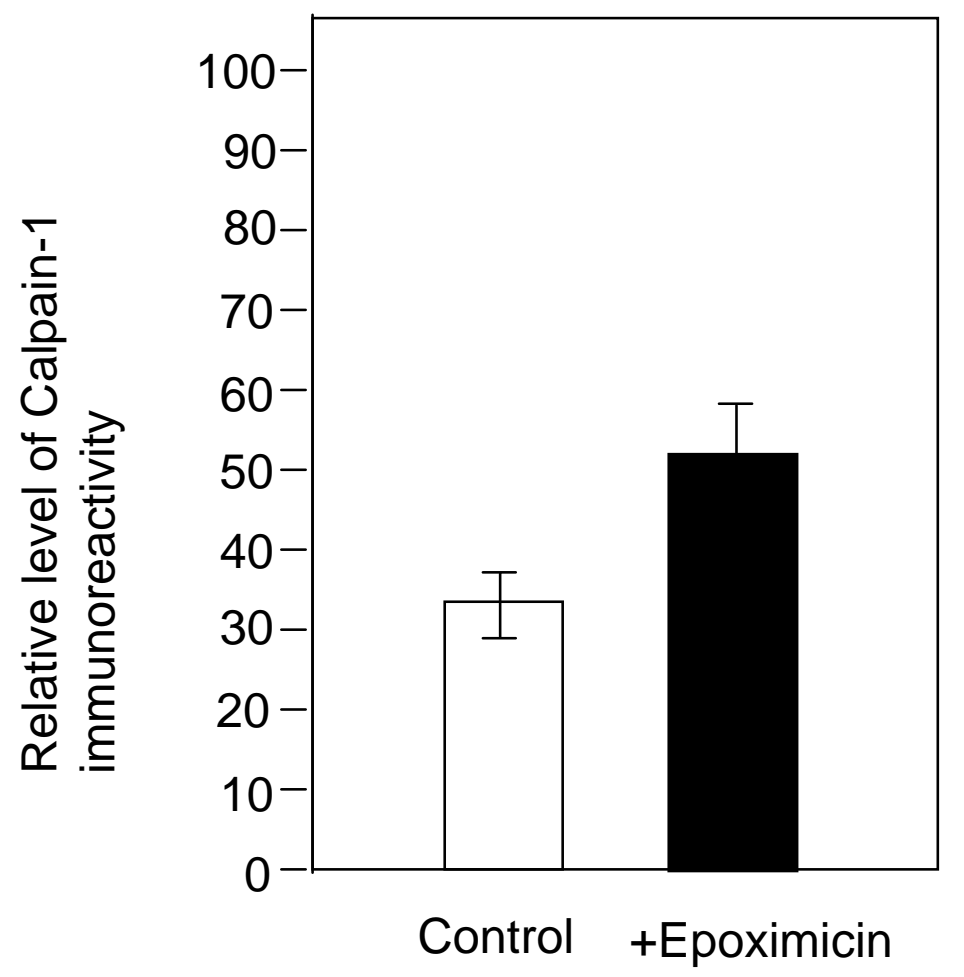

Supplemental Figure S4. ELISA analysis of cultured human primary TM cells for calpain-1 immunoreactivity. Control and inhibition lanes shows $10 \mu \mathrm{g}$ of total protein from primary TM cells with or without inhibition of proteasome using epoximicin as described in methods. Total calpain-1 immunoreactivity was determine using a direct protein loading ELISA assay as described in methods. 